\title{
Some analytical solutions for validation of free surface flow computational codes
}

\section{Marie-Odile Bristeau ${ }^{1}$, Bernard Di Martino ${ }^{1,2}$, Anne Mangeney ${ }^{1,3}$, Jacques Sainte-Marie ${ }^{1}$ and Fabien Souillé ${ }^{1,4}$}

\author{
${ }^{1}$ Inria Paris, 2 rue Simone Iff, CS 42112, 75589 Paris Cedex 12 and Sorbonne Université, Univ. \\ Paris-Diderot SPC, CNRS, Laboratoire Jacques-Louis Lions, LJLL, F-75005 Paris \\ ${ }^{2}$ UMR CNRS 6134 SPE, Université de Corse, Campus Grimaldi, BP 52, 20250 Corte, France \\ ${ }^{3}$ Université de Paris, Institut de Physique du Globe de Paris, 1 rue Jussieu, Paris F-75005, \\ France \\ ${ }^{4}$ EDF R\&D - Laboratoire National d'Hydraulique et Environnement, 6 quai Watier, F-78400 \\ Chatou
}

(Received xx; revised xx; accepted xx)

We present several time dependent analytical solutions for the incompressible Euler system with free surface. These analytical solutions give quantitative descriptions of some physical phenomena, such as water motion or waves on space variable topography, and can be used as reference solutions when validating numerical simulation codes. They concern fluid flows governed by Euler equations with or without hydrostatic hypothesis including wet/dry interface, variable density and wide variety of boundary conditions.

\section{Introduction}

Models arising in fluid dynamics are often based on the Euler equations and are generally difficult to analyze both at the mathematical and numerical level. As a consequence the derivation of simplified models is important. But despite this, the design of efficient/validated numerical schemes for such models remains complex even for the simplified model. The presence of the free surface coupled with the non-linearities complicates the numerical analysis of such models. Even if some discrete stability properties can be proved (consistancy, invariant domains...), some of them e.g. discrete entropy inequalities are hardly accessible and in most of the cases the proof of the convergence of the numerical scheme is out of reach.

A possible approach to validate numerical codes is to compare the results of these codes in configurations for which it is possible to describe the solution exactly using usual functions. There solutions are named analytical solutions. In the literature some examples of analytical solutions for the equations studied here are proposed. One of the most famous concerns a shallow water flow over a parabolic bed (Thacker 1981). An extension of this solution has been proposed by Matskevitch et al. (Matskevich \& Chubarov 2019) and we present here two other extensions. MacDonalds et al. (MacDonald et al. $1995 \mathrm{a}$ b) describe a family of analytical solutions with bottom friction, hydraulic jumps and various open-channel cross-sections. Other series of solutions for hydrostatic free surface Euler equations are proposed by Boulanger et al. (Boulanger et al. 2013) and references therein.

This paper proposes a new serie of analytical solutions that allow to validate the efficiency of the numerical tools developed for the approximation of the incompressible Euler system with free surface. These analytical solutions can be adapted to two- 


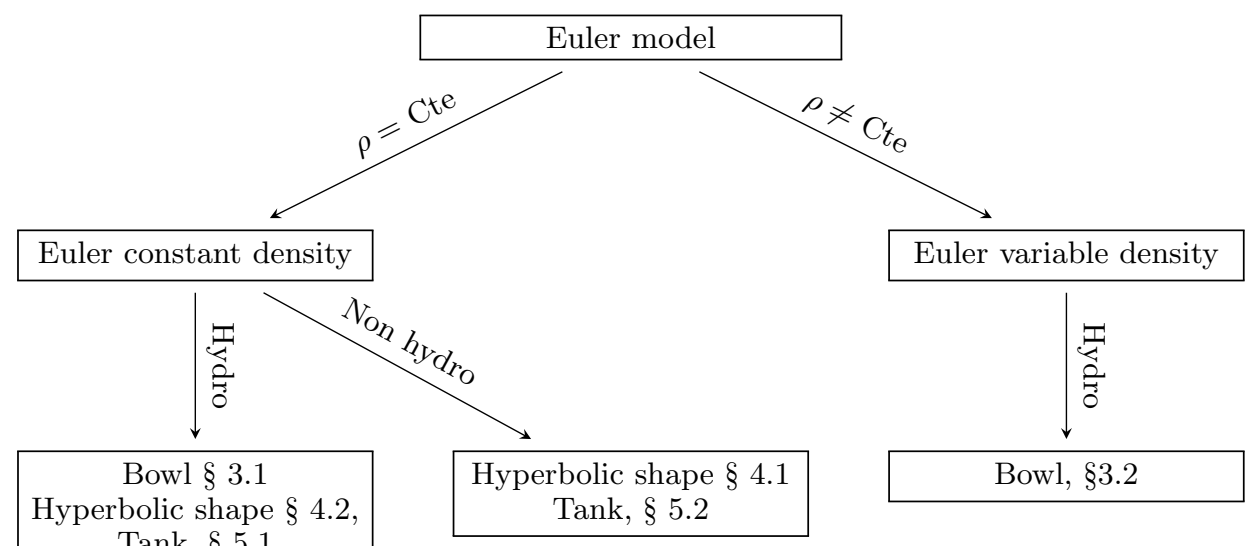

FiguRE 1. Links between the analytical solutions proposed and the models considered. With indication of the shape of the domain (Bowl, Tank, Hyperbolic) and the paragraph where they are presented.

dimensional shallow water type models or to models where the velocity is distributed along the vertical direction. Some of the proposed solutions include

- wet/dry interfaces,

- the hydrostatic assumption,

- a variable density (the density depends on a tracer concentration).

Figure 1 presents a simplified distribution of the proposed analytical solutions and the corresponding model. The indications Bowl, Hyperbolic or Tank refer to the shape of the basin and $\S$ precede the section number to be referred to.

To validate the numerical implementation of the boundary conditions, we have analytical solutions in a cubic tank with open boundaries and in a semi-open domain with hyperbolic topography and open boundary. In such cases we have access to the value of all variables and if necessary all derivatives of these variables at the boundary.

It is important to notice that these analytical solutions correspond to possible physical configurations, even if they are expressed over simple geometrical domains. To compare them to a laboratory experience or a numerical simulation, it is necessary to be able to impose the suitable boundary conditions, what is practicable without difficulties in a numerical simulation but can become more complex in a laboratory experiment.

All the graphics presented is this document represent the exact analytical solutions, but we are able to obtain a good approximation of them by using the Freshkiss3D simulation code (Freshkiss3d 2017) by imposing only the geometry of the domain and the corresponding initial and boundary conditions. With these analytical solutions, it is possible to test simulation codes and to obtain convergence curves when increasing the numerical scheme order and the mesh resolution, see for example (Allgeyer et al. 2019).

This paper is organized as follows. In Section 2 we present the notations and the various forms of the Euler systems used in this paper. In Section 3 we propose two extensions of the solution proposed by Thacker (Thacker 1981) in a parabolic bowl in the case of the hydrostatic Euler model. Firstly an extention of the analytical solution with curved surface in order to have a velocity distributed along the vertical direction. Secondly, an extention of the analytical solution with planar surface including variable density . In Section 4 we consider a domain with a hyperbolic topography for which we can exhibit some solutions for the hydrostatic and the non hydrostatic Euler model. In Section 5 


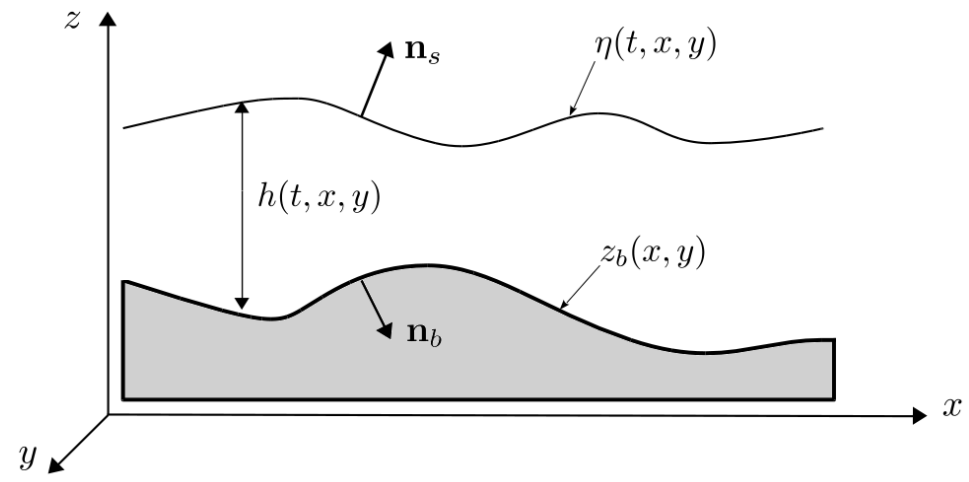

Figure 2. Flow domain with water height $h(t, x, y)$, free surface $\eta(t, x, y)$ and bottom $z_{b}(x, y)$.

we present a cubic tank domain that permits to test the boundary conditions for the hydrostatic and the non hydrostatic Euler model.

\section{The Euler system}

\subsection{Free surface Euler model}

Let us first describe below the mass and momentum equations with associated boundary conditions for which we will propose analytical solutions. We assume in this part that the density is constant. We consider the three-dimensional Euler system describing a free surface gravitational flow moving over a bottom topography $z_{b}(x, y)$ (unit: $\mathrm{m}$ ) with constant density.

$$
\begin{aligned}
& \nabla \cdot \mathbf{U}=0, \\
& \frac{\partial \mathbf{U}}{\partial t}+\nabla \cdot(\mathbf{U} \otimes \mathbf{U})+\frac{1}{\rho_{0}} \nabla p=\mathbf{g}
\end{aligned}
$$

where $\mathbf{U}(t, x, y, z)=(u, v, w)^{T}$ is the velocity (unit: $\left.\mathrm{m} . \mathrm{s}^{-1}\right), p$ is the fluid pressure (unit: $\mathrm{Pa}), \rho_{0}$ is the density (unit: $\mathrm{kg} \cdot \mathrm{m}^{-3}$ ) assumed to be constant and $\mathbf{g}=(0,0,-g)^{T}$ represents the gravity forces (unit: $\mathrm{m} \cdot \mathrm{s}^{-2}$ ). The quantity nabla denotes $\nabla=\left(\frac{\partial}{\partial x}, \frac{\partial}{\partial y}, \frac{\partial}{\partial z}\right)^{T}$. We consider a free surface flow (see Fig. 2), therefore we assume

$$
z_{b}(x, y) \leqslant z \leqslant \eta(t, x, y):=h(t, x, y)+z_{b}(x, y),
$$

with $h(t, x, y)$ the water depth (unit: $\mathrm{m})$.

\subsection{Boundary conditions}

At the free surface, the kinematic boundary condition is

$$
\frac{\partial \eta}{\partial t}+u_{s} \frac{\partial \eta}{\partial x}+v_{s} \frac{\partial \eta}{\partial y}-w_{s}=0
$$

whereas at the bottom we have the non-penetration condition

$$
u_{b} \frac{\partial z_{b}}{\partial x}+v_{b} \frac{\partial z_{b}}{\partial y}-w_{b}=0
$$

where $U_{s}=\left(u_{s}, v_{s}, w_{s}\right)^{T}=(u(t, x, y, \eta), v(t, x, y, \eta), w(t, x, y, \eta))^{T}, U_{b}=\left(u_{b}, v_{b}, w_{b}\right)^{T}=$ $\left(u\left(t, x, y, z_{b}\right), v\left(t, x, y, z_{b}\right), w\left(t, x, y, z_{b}\right)\right)^{T}$ with $\eta=\eta(t, x, y), z_{b}=z_{b}(x, y)$. 
The dynamic boundary condition at the free surface is given by

$$
p_{s}=p(t, x, y, \eta)=p^{a}(t, x, y) .
$$

The system (2.1)-2.5 has to be completed with initial and boundary conditions at the lateral boundaries (inflow, outflow or wall type) that are not detailed here but will be defined for each analytical solution.

\subsection{Hydrostatic models}

Hydrostatic models consist in neglecting the vertical acceleration of the fluid i.e.

$$
\frac{\partial w}{\partial t}+u \frac{\partial w}{\partial x}+v \frac{\partial w}{\partial y}+w \frac{\partial w}{\partial z} \approx 0
$$

Hence, the hydrostatic Euler system writes

$$
\begin{aligned}
& \nabla \cdot \mathbf{U}=0, \\
& \frac{\partial \mathbf{u}}{\partial t}+\nabla_{x, y} \cdot(\mathbf{u} \otimes \mathbf{u})+\frac{\partial(\mathbf{u} w)}{\partial z}+\frac{1}{\rho_{0}} \nabla_{x, y} p=0, \\
& \frac{\partial p}{\partial z}=-\rho_{0} g
\end{aligned}
$$

where $\mathbf{u}(t, x, y, z)=(u, v)^{T}$ is the horizontal velocity, $\nabla_{x, y}$ correspond to the projection of $\nabla$ on the horizontal plane, i.e. $\nabla_{x, y}=\left(\frac{\partial}{\partial x}, \frac{\partial}{\partial y}\right)^{T}$. These equations are completed with the boundary conditions $2.3,2.4$ and $(2.5)$. The vertical momentum equation leads then to the hydrostatic pressure

$$
p(t, x, y, z)=p^{a}(t, x, y)+\rho_{0} g(\eta-z) .
$$

These hydrostatic models are very often used for the study of geophysical flows, see Brenier (1999); Grenier (1999) such as landslides, glaciers or tsunamis (e. g. Fernandez-Nieto et al. (2016); Allgeyer et al. (2019)) for justifications of such models.

\subsection{Passive tracer or variable density}

We can consider that the fluid contains a passive tracer $\phi(t, x, y, z)$ governed by a transport equation

$$
\frac{\partial \phi}{\partial t}+\mathbf{U} \cdot \nabla \phi=0
$$

Equation 2.11) with $\rho=\rho_{0}$ implies that this tracer has no impact on the flow.

But we can also consider a fluid where the density is a function of the tracer concentration $\phi$ i.e. $\rho=\rho(\phi)$ (typically, $\phi$ can represent the temperature or the salinity of the fluid). $\rho(\phi)$ being a given function, the hydrostatic Euler system with variable density writes

$$
\begin{aligned}
& \nabla \cdot \mathbf{U}=0 \\
& \frac{\partial \rho}{\partial t}+\nabla \cdot(\rho \mathbf{U})=0 \\
& \frac{\partial(\rho \mathbf{u})}{\partial t}+\nabla_{x, y} \cdot(\rho \mathbf{u} \otimes \mathbf{U})+\nabla_{x, y} p=0, \\
& \frac{\partial p}{\partial z}=-\rho g .
\end{aligned}
$$

The system (2.12)-(2.14) is completed with the boundary conditions $(2.3), 2.4), 2.5)$. 
Notice that Eqs. 2.12, 2.13 with $\rho=\rho(\phi)$ give (2.11). But thought the density variations in Eqs. 2.14), 2.15) the variations of the tracer concentration $\phi(t, x, y, z)$ modify the flow.

\section{Parabolic bowl}

This first set of analytical solutions we propose are extensions of the solutions introduced by Thacker (1981). The solution initially proposed by Thacker corresponds to the solution of shallow water equations i.e. an hydrostatic flow where the horizontal velocity $(u, v)$ does not depend on the vertical coordinate $z$. Thacker's approach requires a parabolic shape of the basin and its solution implies to treat wet/dry interfaces. We propose here two extensions of Thacker's solutions, the first one having a velocity distributed along the vertical axis, and the second one dealing with a variable density flow. For both of them, the bottom topography is given by

$$
z_{b}(x, y)=\alpha \frac{r^{2}}{2}
$$

with $\alpha>0$ and $r=\sqrt{x^{2}+y^{2}}$.

\subsection{Hydrostatic Euler model and parabolic topography}

We propose here an extension of the Thacker's solutions with curved surface Thacker (1981) where components of the velocity are functions of the $z$ variable. The following proposition gives an analytical solution for the hydrostatic Euler model presented in Section 2.3 ,

Proposition 1. For some $t_{0} \in \mathbb{R},(\alpha, \beta, \gamma) \in \mathbb{R}_{+*}^{3}$, such that $\gamma<1$ let us consider the functions $h, u, v, w, p$ defined for $t \geqslant t_{0}$ by

$$
\begin{aligned}
h(t, x, y)= & \max \left\{0, \frac{1}{r^{2}} f\left(\frac{r^{2}}{\gamma \cos (\omega t)-1}\right)\right\} \\
u(t, x, y, z)= & x\left(\beta\left(z-z_{b}-\frac{h}{2}\right)+\frac{\omega \gamma \sin (\omega t)}{2(1-\gamma \cos (\omega t))}\right) \\
v(t, x, y, z)= & y\left(\beta\left(z-z_{b}-\frac{h}{2}\right)+\frac{\omega \gamma \sin (\omega t)}{2(1-\gamma \cos (\omega t))}\right) \\
w(t, x, y, z)= & -\beta\left(z-z_{b}\right)^{2}+\left(\beta h-2 \Gamma-\beta \alpha r^{2}+\frac{\beta}{2}\left(x \frac{\partial h}{\partial x}+y \frac{\partial h}{\partial y}\right)\right)\left(z-z_{b}\right) \\
& -\frac{\alpha r^{2}}{2}(\beta h-2 \Gamma) \\
p(t, x, y, z)= & g(\eta-z)
\end{aligned}
$$

with $\omega=\sqrt{4 \alpha g}, \Gamma=-\frac{\omega \gamma \sin (\omega t)}{2 \Lambda}, \Lambda=\gamma \cos (\omega t)-1$ and the function $f$ given by

$$
f(z)=-\frac{4 g}{\beta^{2}}+\frac{2}{\beta^{2}} \sqrt{4 g^{2}+c z+\beta^{2} \alpha g\left(\gamma^{2}-1\right) z^{2}},
$$

$c$ being a nonpositive constant.

Then $h, u, v, w$ and $p$ as defined previously satisfy the $3 d$ hydrostatic Euler system (2.7)(2.8) completed with the boundary conditions (2.3), 2.4) and (2.5) with $p^{a}=$ cst. 


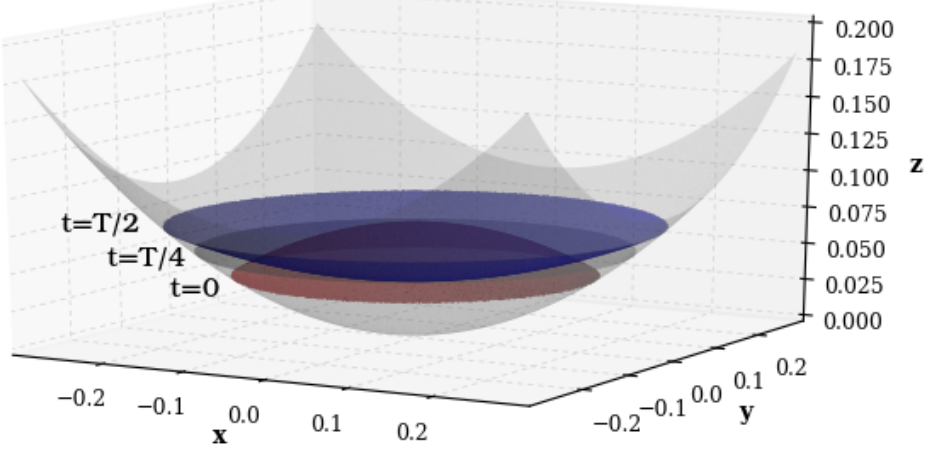

Figure 3. Analytic solution for 3D Axisymmetrical parabolic bowl (see prop 1): free surface at $t=0$ (red), $t=T / 4$ (dark grey), $t=T / 2$ (blue), with the period $T$ defined by $T=2 \pi / \omega$ and for parameters set to $h_{0}=1 \mathrm{~m}, \alpha=2 \mathrm{~m}^{-1}, \beta=1 \mathrm{~m}^{-1} \cdot \mathrm{s}^{-1}, \gamma=0.3, c=-1 \mathrm{~s}^{-4}, L=1 \mathrm{~m}$.

Proof. The construction of this solution is inspired by the Thacker work (Thacker 1981). In order to extend the Thacker solution, we first observe that

$$
\int_{z_{b}}^{z_{b}+h} \varphi(t, x, y)\left(z-z_{b}-\frac{h}{2}\right) \mathrm{d} z=0,
$$

for all function $\varphi(t, x, y)$. Thus, if we add a term having the form

$$
\varphi(t, x, y)\left(z-z_{b}-\frac{h}{2}\right)
$$

to the components of the horizontal velocity field, this does not modify a large number of the properties such as the mass conservation. However, some adjustments are necessary to meet all the requirements imposed by Eqs. (2.1)- 2.2 and allowing to precise the expression of $\varphi(t, x, y)$. Asymptotically, when $\beta$ goes to zero, the proposed analytical solution converges to the one proposed by Thacker. The proof of Prop. 1 is based on the verification that the proposed expressions for $h, u, v, w$ and $p$ are solutions of the equations

$$
\begin{aligned}
& \frac{\partial h}{\partial t}+\frac{\partial}{\partial x} \int_{z_{b}}^{z_{b}+h} u \mathrm{~d} z+\frac{\partial}{\partial y} \int_{z_{b}}^{z_{b}+h} v \mathrm{~d} z=0, \\
& \frac{\partial u}{\partial t}+u \frac{\partial u}{\partial x}+v \frac{\partial u}{\partial y}+w \frac{\partial u}{\partial z}+g \frac{\partial\left(h+z_{b}\right)}{\partial x}=0 \\
& \frac{\partial v}{\partial t}+u \frac{\partial v}{\partial x}+v \frac{\partial v}{\partial y}+w \frac{\partial v}{\partial z}+g \frac{\partial\left(h+z_{b}\right)}{\partial x}=0
\end{aligned}
$$

where Eq. 3.9 comes from an integration over the vertical axis of the divergence free condition coupled with the two kinematic boundary conditions $(2.3)$ and $(2.4)$.

The details of the computation are given only for equation (3.9). A more complete computation will be carried out for the two-dimensional case in the proof of Corollary 1 . From (3.1) and the definition of $f(z)$ given in (3.7), we can verify that on the set where $h(t, x, y)>0$ we have

$$
\frac{\partial h}{\partial t}=\frac{\gamma \omega \sin (\omega t)}{(\gamma \cos (\omega t)-1)^{2}} f^{\prime}(),
$$


with the notation $f()=f\left(\frac{r^{2}}{\gamma \cos (\omega t)-1}\right)$. From equation (3.3), we obtain

$$
\int_{z_{b}}^{z_{b}+h} u \mathrm{~d} z=\frac{h x \omega \gamma \sin (\omega t)}{2(1-\gamma \cos (\omega t))}, \quad \int_{z_{b}}^{z_{b}+h} v \mathrm{~d} z=\frac{h y \omega \gamma \sin (\omega t)}{2(1-\gamma \cos (\omega t))} \text {. }
$$

Since

$$
\frac{\partial h}{\partial x}=-\frac{2 x f()}{r^{4}}+\frac{2 x f^{\prime}()}{\left.r^{2}(\gamma \cos (\omega t)-1)\right)}
$$

we have

$$
\frac{\partial}{\partial x} \int_{z_{b}}^{z_{b}+h} u \mathrm{~d} z=\frac{\omega \gamma \sin (\omega t)}{2(1-\gamma \cos (\omega t))}\left[\frac{f()}{r^{2}}-\frac{2 x^{2} f()}{r^{4}}+\frac{2 x^{2} f^{\prime}()}{r^{2}}\right],
$$

and

$$
\frac{\partial}{\partial y} \int_{z_{b}}^{z_{b}+h} v \mathrm{~d} z=\frac{\omega \gamma \sin (\omega t)}{2(1-\gamma \cos (\omega t))}\left[\frac{f()}{r^{2}}-\frac{2 y^{2} f()}{r^{4}}+\frac{2 y^{2} f^{\prime}()}{r^{2}}\right] .
$$

We deduce that

$$
\frac{\partial}{\partial x} \int_{z_{b}}^{z_{b}+h} u \mathrm{~d} z+\frac{\partial}{\partial y} \int_{z_{b}}^{z_{b}+h} v \mathrm{~d} z=\frac{\omega \gamma \sin (\omega t)}{(1-\gamma \cos (\omega t))^{2}} f^{\prime}() .
$$

and Eq. 3.9 is satisfied.

In Figure 3 , the shape of the free surface of the analytical solution is plotted at different time instants. In (Allgeyer et al. 2019), convergence curves towards the analytical solution are obtained with the numerical code (Freshkiss3d 2017).

In Figures 4, we represented an axial section of the analytical solution at four time instants $(t=0, T / 6, T / 3, T / 2)$ for a parameters set $h_{0}=1 \mathrm{~m}, \alpha=2 \mathrm{~m}^{-1}, \beta=$ $1 \mathrm{~m}^{-1} \cdot \mathrm{s}^{-1}, \gamma=0.3, c=-1 \mathrm{~s}^{-4}, L=1 \mathrm{~m}$. The arrows represent the velocity field and the color shading gives the velocity norm. 

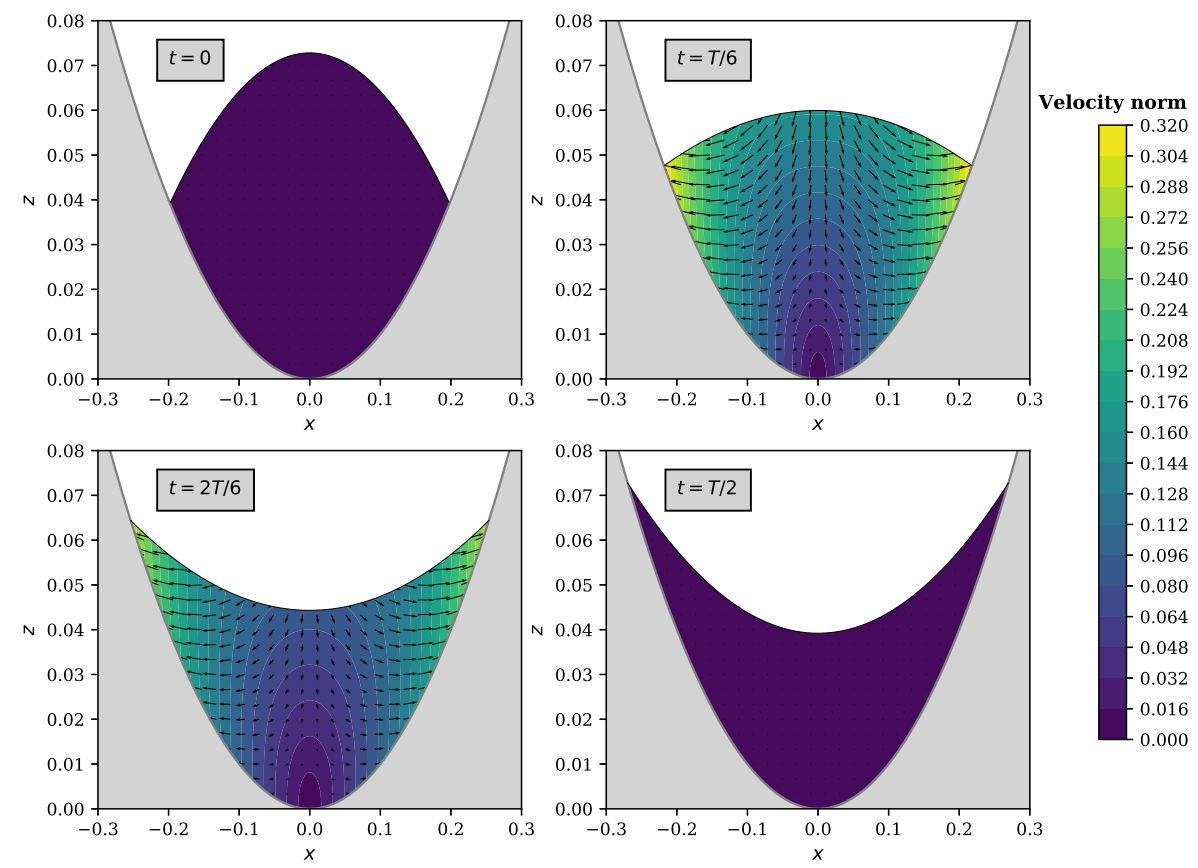

Figure 4. Analytic solution for 3D Axisymmetrical parabolic bowl (see prop 1): velocity field and norm at $t=0, T / 6,2 T / 6, T / 2$, in $(x, y=0, z)$ slice plane with the period $T$ defined by $T=2 \pi / \omega$ and for parameters set to $h_{0}=1 \mathrm{~m}, \alpha=2 \mathrm{~m}^{-1}, \beta=1 \mathrm{~m}^{-1} \cdot \mathrm{s}^{-1}, \gamma=0.3, c=-1 \mathrm{~s}^{-4}$, $L=1 \mathrm{~m}$.

The analytical solution proposed in Prop. 1 can be also expressed in a two dimensional $(x, z)$-domain and the following corollary holds.

Corollary 1. The analytical solution depicted in Prop. 1 can be written in the $2 d$ case. With obvious notations and $\beta>0$, we choose $\gamma \leqslant 2 g /(\beta \omega), c>0$, and we consider the functions $h, u, w, p$ defined for $t \geqslant t_{0}$ by

$$
\begin{aligned}
& h(t, x)=\max \left\{0, f\left(x-\frac{\gamma}{\omega} \sin (\omega t)\right)\right\} \\
& u(t, x, z)=\beta\left(z-z_{b}-\frac{h}{2}\right)+\gamma \cos (\omega t) \\
& w(t, x, z)=\alpha \beta x z-\frac{\alpha^{2} \beta}{2} x^{3}-\frac{\alpha \beta}{2} x h(t, x)+\frac{\beta}{2}\left(z-\frac{\alpha}{2} x^{2}\right) \frac{\partial h}{\partial x}+\alpha \gamma x \cos (\omega t), \\
& p(t, x, z)=g(\eta-z)
\end{aligned}
$$

with $\omega=\sqrt{\alpha g}$, a bottom topography defined by

$$
z_{b}(x)=\frac{\alpha}{2} x^{2}
$$

and a function $f$ given by

$$
f(z)=-\frac{4 g}{\beta^{2}}+\frac{2}{\beta^{2}} \sqrt{4 g^{2}+\beta^{2} c^{2}-\beta^{2}(\omega z+c)^{2}} .
$$

Then $h, u, w, p$ as defined previously satisfy the 2d hydrostatic Euler system completed with the boundary conditions (2.3), 2.4 and 2.5 with $p^{a}=$ cst. 
Proof. It is enough to verify that the expressions of $u, w$, and $h$ are solutions of the following equations

$$
\begin{aligned}
& \frac{\partial h}{\partial t}+\frac{\partial}{\partial x} \int_{z_{b}}^{z_{b}+h} u(x, z) \mathrm{d} z=0, \\
& \frac{\partial u}{\partial t}+u \frac{\partial u}{\partial x}+w \frac{\partial u}{\partial z}+g \frac{\partial\left(h+z_{b}\right)}{\partial x}=0,
\end{aligned}
$$

where $w$ is then given thanks to the incompressibility condition by

$$
w(t, x, z)=-\frac{\partial}{\partial x} \int_{z_{b}}^{z} u d z .
$$

From 3.12 and the definition of $f(z)$ given in 3.16 , we can verify that on the set where $h(t, x)>0$ we have

$$
\frac{\partial h}{\partial t}=-\gamma \cos (\omega t) f^{\prime}\left(x-\frac{\gamma}{\omega} \sin (\omega t)\right)
$$

From Eq. (3.13) and using Eq. (3.8) we find

$$
\int_{z_{b}}^{z_{b}+h} u(x, z) \mathrm{d} z=h \gamma \cos (\omega t)
$$

And since

Eq. (3.17) is satisfied.

$$
\frac{\partial h}{\partial x}=f^{\prime}\left(x-\frac{\gamma}{\omega} \sin (\omega t)\right)
$$

In order to verify Eq. (3.18), we start from the definition of $u(t, x, z)$ leading to

$$
\frac{\partial u}{\partial t}=\frac{\beta \gamma \cos (\omega t)}{2} f^{\prime}()-\gamma \omega \sin (\omega t)
$$

and

$$
u \frac{\partial u}{\partial x}=-\beta\left(4 \alpha x+\frac{1}{2} f^{\prime}()\right)\left[\beta\left(z-2 \alpha x^{2}-\frac{1}{2} f()\right)+\gamma \cos (\omega t)\right],
$$

with the notation $f()=f\left(x-\frac{\gamma}{\omega} \sin \omega t\right)$. Moreover, we have

$$
w \frac{\partial u}{\partial z}=\beta\left(4 \alpha \beta x z-8 \alpha^{2} \beta x^{3}-2 \alpha \beta x f()+\beta\left(\frac{z}{2}-\alpha x^{2}\right) f^{\prime}()+4 \alpha \gamma x \cos (\omega t)\right) .
$$

And the pressure term gives

$$
g \frac{\partial\left(h+z_{b}\right)}{\partial x}=g f^{\prime}()+4 g \alpha x .
$$

Suming the last four expressions, all the terms without $f()$ or $f^{\prime}()$ are equal to $4 g \alpha x$ whereas the terms containing $f()$ only are equal to zero. The terms containing only $f^{\prime}()$ are equal to $g-\frac{\beta \gamma}{2} \cos \omega t$, and the terms containing $f() \cdot f^{\prime}()$ equals $\frac{\beta^{2}}{4}$. Now, we have to observe that equation 3.16 gives

$$
f^{\prime}\left(x-\frac{\gamma}{\omega} \sin (\omega t)\right)=-\frac{2 \omega(\omega x-\gamma \sin (\omega t))}{\sqrt{4 g^{2}-2 c \beta^{2}-\beta^{2}\left(\omega^{2} x-2 \gamma \omega \sin (\omega t)+\gamma^{2} \sin ^{2}(\omega t)\right)}} .
$$

Since

$$
f(\xi) \cdot f^{\prime}(\xi)=-\frac{4 g}{\beta^{2}} f^{\prime}()-\frac{4 \omega^{2}}{\beta^{2}} \xi
$$




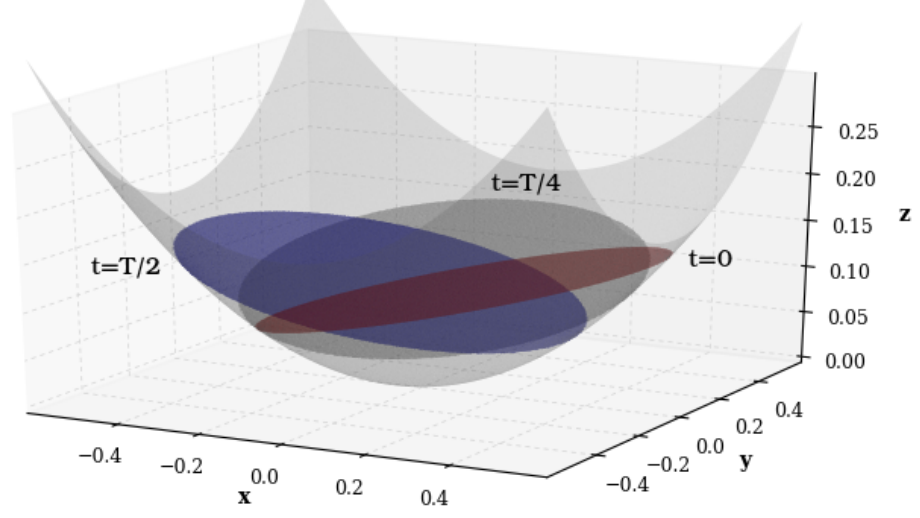

FiguRE 5. Analytical solution of radially-symmetrical parabolic bowl with variable density (see prop. 2), 3D planar surface: free surface at $t=0$ (red), $t=T / 4$ (dark grey), $t=T / 2$ (blue), with the period $T$ defined by $T=2 \pi / \omega$ and for parameters set to $\eta=0.1 \mathrm{~m}, h_{0}=0.1 \mathrm{~m}, a=1$, $\alpha=1 \mathrm{~m}^{-1}, L=4 \mathrm{~m}$.

the expressions of $h, u$ and $w$ inserted in Eq. (3.18) leads to $2 g \alpha x-\omega^{2} x$, and this term vanishes under the condition $\omega=\sqrt{2 g \alpha}$.

\subsection{Hydrostatic Euler equation with variable density}

The analytical solution proposed here is based on the analytical solution presented by Thacker (Thacker 1981) when the free surface remains planar. The initial Thacker's solution is valid for the 2d Saint-Venant system and also for the $3 \mathrm{~d}$ incompressible and hydrostatic Euler system with constant density. The extension proposed in the following proposition gives an analytical solution for the hydrostatic Euler system with variable density (2.12)-(2.14).

Proposition 2. For any nonnegative function $\rho: s \mapsto \rho(s)$ and for some $\left(\alpha, \eta, a, h_{0}\right) \in \mathbb{R}^{2} \times \mathbb{R}_{+}^{2}$, let us consider the functions $h, u, v, w, p, \phi$ defined for $(x, y) \in[-L / 2, L / 2]^{2}, t \geqslant t_{0}$ by

$$
\begin{aligned}
& h(t, x, y)=\max \left\{0, h_{0}-\alpha \frac{(x-\eta \cos (\omega t))^{2}+(y-\eta \sin (\omega t))^{2}}{2}\right\}, \\
& u(t, x, y, z)=-\eta \omega \sin (\omega t) \\
& v(t, x, y, z)=\eta \omega \cos (\omega t) \\
& w(t, x, y, z)=-\alpha \eta \omega(x \sin (\omega t)-y \cos (\omega t)) \\
& p(t, x, y, z)=p^{a}(t)+\int_{z}^{h+z_{b}} \rho\left(\phi\left(t, x, y, z_{1}\right)\right) d z_{1}, \\
& \phi(t, x, y, z)=a\left(h+z_{b}-z\right),
\end{aligned}
$$

with $\omega=\sqrt{\alpha g}$. We recall that the topography is given by $z_{b}(x, y)=\frac{\alpha}{2} r^{2}$. Then $h, u, v, w, p$ and $\phi$ as defined previously satisfy the $3 d$ hydrostatic Euler system with variable density (2.12)-2.14 completed with the kinematic boundary conditions (2.3)-2.4.

A plot of the analytical solution (in the plane $y=0$ ) at several time instants is given in Fig. 6 where we can observe that the density isovalue lines remain parallel to the planar surface.

Situations where $\frac{\partial \rho}{\partial z} \geqslant 0$ can be encountered in practice (upwellings, Rayleigh-Bénard 

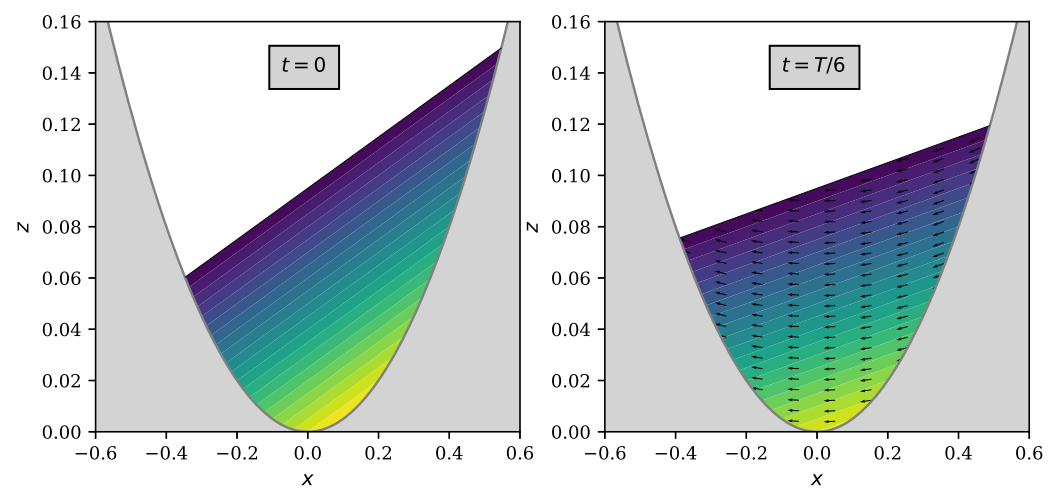

Tracer
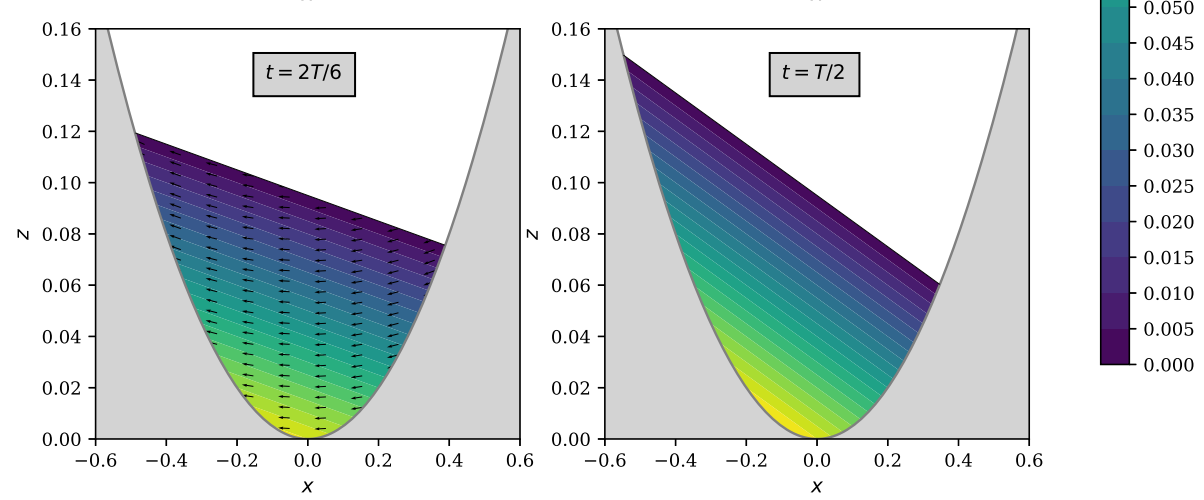

Figure 6. Analytical solution of radially-symmetrical parabolic bowl with variable density (see prop. 2): free surface, velocity vectors and tracer for $t=0, T / 6,2 T / 6, T / 2$, in $(x, y=0, z)$ slice plane with the period $T$ defined by $T=2 \pi / \omega$ and for parameters set to $\eta=0.1 \mathrm{~m}, h_{0}=0.1 \mathrm{~m}$, $a=1, \alpha=1 \mathrm{~m}^{-1}, L=4 \mathrm{~m}$.

instabilities,...). The analytical solutions given in prop. 2 exhibit situations where $\frac{\partial \rho}{\partial z}$ remains nonnegative along the time, of course, such solutions are unstable in the sense that they cannot be reproduced neither by laboratory experiments nor captured at the discrete level.

Proof. Starting from Thacker's solution with constant density (Thacker 1981), we have only to verify that the chosen expression for the density $\rho(t, x, y, z)=a(\eta(t, x, y)-z)$ does not modify this solution. We have $\partial_{z} p=-\rho(t, x, y, z) g$ due to the hydrostatic hypothesis. If we note $\eta=\eta(t, x, y)=h(t, x, y)+z_{b}(x, y), \rho(t, x, y, z)=\phi(\eta(t, x, y)-z)$ 
12 M.-O. Bristeau, B. Di Martino, A. Mangeney, J. Sainte-Marie and F. Souillé and $p(t, x, y, \eta(t, x, y))=p^{a}(t)$, for $\phi \in C^{1}(\mathbb{R})$ we have

$$
\begin{aligned}
\nabla_{x, y} p & =g \nabla_{x, y} \int_{z}^{\eta} \rho(t, x, y, \xi) \mathrm{d} \xi \\
& =g \nabla_{x, y} \int_{z}^{\eta} \phi(\eta-\xi) \mathrm{d} \xi \\
& =g \int_{z}^{\eta} \nabla_{x, y} \phi(\eta-\xi) \mathrm{d} \xi+g \phi(0) \nabla_{x, y} \eta \\
& =g \int_{z}^{\eta} \phi^{\prime}(\eta-\xi) \nabla_{x, y} \eta \mathrm{d} \xi+g \phi(0) \nabla_{x, y} \eta \\
& =g \nabla_{x, y} \eta \int_{\eta-z}^{0} \phi^{\prime}(s) \mathrm{d} s+g \phi(0) \nabla_{x, y} \eta \quad \text { with } s=\eta-\xi \\
& =g \phi(\eta-z) \nabla_{x, y} \eta \\
& =g \rho(t, x, y, z) \nabla_{x, y} \eta .
\end{aligned}
$$

If we consider the momentum equation 2.14, using the previous evaluation of the pressure term, we can conclude that $u$ is solution of equation $(2.8)$ that corresponds to the momentum equation with constant density.

But generally, this expression of the density does not verify the conservation equation 2.13

$$
\partial_{t} \rho+\nabla \cdot(\rho U)=0
$$

In the proposed analytical solution, since $u$ and $w$ are independent of $x$ and $z$, then we have

$$
\begin{aligned}
\partial_{t} \rho+\mathbf{u} \cdot \nabla_{x, y} \rho+w \partial_{z} \rho & =\phi^{\prime}(\eta-z) \partial_{t} \eta+\phi^{\prime}(\eta-z) \mathbf{u} \cdot \nabla_{x, y} \eta-\phi^{\prime}(\eta-z) w \\
& =\phi^{\prime}(\eta-z)\left(\partial_{t} \eta+\mathbf{u} \cdot \nabla_{x, y} \eta-w\right)=0
\end{aligned}
$$

since at the free surface the kinematic boundary condition gives $\partial_{t} \eta+\mathbf{u} \cdot \nabla_{x, y} \eta-w=0$.

The analytical solution depicted in prop. 2 cadmits a $2 \mathrm{~d}$ version.

Corollary 2. With obvious notations, the functions $h, u, w, p, \phi$ defined for $t \geqslant t_{0}$ by

$$
\begin{aligned}
& h(t, x)=\max \left\{0, h_{0}-\alpha \frac{(x-\eta \cos (\omega t))^{2}}{2}\right\}, \\
& u(t, x, z)=-\eta \omega \sin (\omega t), \\
& w(t, x, z)=-\alpha x \eta \omega \sin (\omega t), \\
& p(t, x, z)=p^{a}(t)+\int_{z}^{h+z_{b}} \rho\left(\phi\left(t, x, z_{1}\right)\right) d z_{1}, \\
& \phi(t, x, z)=a(\eta-z),
\end{aligned}
$$

with a bottom topography defined by $z_{b}(x)=\alpha x^{2} / 2$ and with the kinematic boundary conditions (2.3)-(2.4) satisfy the 2d version of the hydrostatic Euler system with variable density 2.12)-2.15) for any $\left(a, \alpha, \eta, h_{0}\right) \in \mathbb{R}^{3} \times \mathbb{R}_{+}$.

Proof. The proof is the same as for Prop. 2 and is not detailed here. 


\section{Hyperbolic topography}

In this section, we are interested in characterizing the shallow water analytical solutions of the Euler system. More precisely, we show that under a reasonable hypothesis, we can find all the solutions of Euler free surface equations whose horizontal velocity does not depend on $z$. More precisely, we exhibit the analytical solutions of the system (2.1)-(2.2) completed with the boundary conditions $(2.3),(2.4)$ and $(2.5)$ having a velocity field of the form $u=\bar{u}(t, x, y)$ and $v=\bar{v}(t, x, y)$. Moreover, it is possible to deduce the shallow water analytical solutions of the hydrostatic Euler equations (2.7)- 2.8]. At the numerical level, these solutions give access to analytical solutions of the free surface Euler equations with an open boundary and wet/dry interfaces.

\subsection{Non hydrostatic Euler equations}

Proposition 3. For some $\left(\alpha, \beta, c_{0}\right) \in \mathbb{R}^{+3},\left(b_{0}, t_{0}\right) \in \mathbb{R}^{2}, t_{1} \in \mathbb{R}_{+}^{*}, \theta \in[0,2 \pi]$, let us consider the functions $h, u, v, w$ and $p$ defined for $t \geqslant t_{0},(x, y) \in \mathbb{R}^{+2}$ and $z \in$ $\left[z_{b}(x, y), \eta(t, x, y)\right]$ by

$$
\begin{aligned}
& h(t, x, y)=\max \left\{0, \alpha f(t)-b_{0}-z_{b}(x, y)\right\}, \\
& u(t, x, y, z)=f(t)(x \cos \theta+y \sin \theta+\beta) \cos \theta, \\
& v(t, x, y, z)=f(t)(x \cos \theta+y \sin \theta+\beta) \sin \theta, \\
& w(t, x, y, z)=-f(t)\left(z+b_{0}\right), \\
& p(t, x, y, z)=p^{a, 0}(t)+f^{2}(t)\left(\eta^{2}-z^{2}\right)+\left(2 b_{0} f^{2}(t)+g\right)(\eta-z),
\end{aligned}
$$

where $f(t)=1 /\left(t-t_{0}+t_{1}\right), p^{a, 0}(t)$ is a given function and the topography is defined by

$$
z_{b}(x, y)=\frac{c_{0}}{x \cos \theta+y \sin \theta+\beta}-b_{0} .
$$

Then $h, u, v$ and $p$ as defined previously satisfy the Euler system (2.1)-(2.2) completed with the boundary conditions 2.3, 2.4 and 2.5.

REMark 1. In Prop. 3, we have to deal with an open boundary, the associate boundary conditions to impose can be obtained considering the value of the analytical expressions of the solution on the corresponding domain boundary.

Proof. The proof relies on simple computations that are detailed hereafter. For the sake of simplicity, the computations are carried out in $2 \mathrm{~d}(x, z)$ corresponding to $\theta=0$.

Assuming $u=\bar{u}(t, x)$, the divergence free condition (2.1) coupled with (2.4) gives

$$
w=-z \frac{\partial \bar{u}}{\partial x}+\frac{\partial\left(z_{b} \bar{u}\right)}{\partial x}
$$

and the two components of 2.2 give

$$
\begin{aligned}
& \frac{\partial \bar{u}}{\partial t}+\bar{u} \frac{\partial \bar{u}}{\partial x}+\frac{\partial p}{\partial x}=0 \\
& -z\left(\frac{\partial^{2} \bar{u}}{\partial x \partial t}+\bar{u} \frac{\partial^{2} \bar{u}}{\partial x^{2}}-\left(\frac{\partial \bar{u}}{\partial x}\right)^{2}\right)+\frac{\partial^{2}\left(z_{b} \bar{u}\right)}{\partial x \partial t}+\bar{u} \frac{\partial^{2}\left(z_{b} \bar{u}\right)}{\partial x^{2}}-\frac{\partial \bar{u}}{\partial x} \frac{\partial\left(z_{b} \bar{u}\right)}{\partial x}+\frac{\partial p}{\partial z}=-g \cdot(4.3)
\end{aligned}
$$

From Eqs. 4.2, 4.3 and 2.5, it comes that the pressure $p$ satisfies

$$
p(t, x, \eta)=p^{a, 0}(t), \quad \text { and } \quad \frac{\partial^{2} p}{\partial x \partial z}=\frac{\partial^{3} p}{\partial z^{3}}=0,
$$


14 M.-O. Bristeau, B. Di Martino, A. Mangeney, J. Sainte-Marie and F. Souillé

therefore the pressure $p$ has necessarily the form

$$
\bar{p}=p^{a, 0}(t)+\frac{a(t)}{2}\left(\eta^{2}-z^{2}\right)+b(t)(\eta-z),
$$

where $a=a(t)$ and $b=b(t)$ are two functions to be determined.

Hence the shallow water solutions of the incompressible Euler system with free surface are characterized by

$$
\begin{aligned}
& \frac{\partial h}{\partial t}+\frac{\partial(h \bar{u})}{\partial x}=0 \\
& \frac{\partial \bar{u}}{\partial t}+\bar{u} \frac{\partial \bar{u}}{\partial x}+(a(t) \eta+b(t)) \frac{\partial \eta}{\partial x}=0, \\
& \frac{\partial^{2} \bar{u}}{\partial x \partial t}+\bar{u} \frac{\partial^{2} \bar{u}}{\partial x^{2}}-\left(\frac{\partial \bar{u}}{\partial x}\right)^{2}=-a(t), \\
& \frac{\partial^{2}\left(z_{b} \bar{u}\right)}{\partial x \partial t}+\bar{u} \frac{\partial^{2}\left(z_{b} \bar{u}\right)}{\partial x^{2}}-\frac{\partial \bar{u}}{\partial x} \frac{\partial\left(z_{b} \bar{u}\right)}{\partial x}=b(t)-g .
\end{aligned}
$$

Now subtracting to Eq. (4.7) the derivative of Eq. (4.6) w.r.t. the variable $x$ gives

$$
2\left(\frac{\partial \bar{u}}{\partial x}\right)^{2}=a(t)-\frac{\partial}{\partial x}\left((a(t) \eta+b(t)) \frac{\partial \eta}{\partial x}\right) .
$$

Likewise, substracting Eq. 4.7 multiplied by $z_{b}$ to Eq. 4.8 gives

$$
\frac{\partial \bar{u}}{\partial t} \frac{\partial z_{b}}{\partial x}+\bar{u}^{2} \frac{\partial^{2} z_{b}}{\partial x^{2}}+\bar{u} \frac{\partial \bar{u}}{\partial x} \frac{\partial z_{b}}{\partial x}+a(t) z_{b}=b(t)-g .
$$

The solution $\eta=\eta(t, x)$ of Eq. (4.6) can be obtained explicitly if we assume $a(t)>0$ and then $h(t, x)$ is given by

$$
h(t, x)=-z_{b}(x)-\frac{b(t)}{a(t)}+\frac{1}{a(t)} \sqrt{a(t)\left(F_{1}(t)-\bar{u}^{2}-2 \int^{x} \partial_{t} \bar{u} d s\right)}
$$

where $t \mapsto F_{1}(t)$ is any function such that $F_{1}(t) \geqslant \bar{u}^{2}+2 \int^{x} \partial_{t} \bar{u} d s$.

A solution of the derivative of equation 4.7 w.r.t. the variable $x$ is given by

$$
\bar{u}(t, x)=\frac{u_{1}(x)}{t-t_{0}+t_{1}} .
$$

Let us assume that 4.11 holds true. Inserting 4.11 into Eq. 4.5), we can write this equation formally under the form

$$
\frac{\partial\left(h u_{1}\right)}{\partial x}+\frac{t-t_{0}+t_{1}}{u_{1}} \frac{\partial\left(h u_{1}\right)}{\partial t}=0
$$

then we apply the characteristic method following $t^{\prime}(x)=\frac{t-t_{0}+t_{1}}{u_{1}}$ leading to $t(x)=$ $\left(t-t_{0}+t_{1}\right) \int^{x} \frac{1}{u_{1}(s)} d s$ and we can exhibit a formal expression of $h(x, t)$ under the form

$$
h(t, x)=\frac{F_{0}\left(\left(t-t_{0}+t_{1}\right) e^{-\int^{x} \frac{d s}{u_{1}(s)}}\right)}{u_{1}(x)},
$$

where $\xi \mapsto F_{0}(\xi)$ is any function.

Likewise, inserting the expression of $\bar{u}$ given by 4.11 into Eq. 4.10 gives another 
expression for $h(t, x)$ with

$h(t, x)=-z_{b}(x)-\frac{b(t)}{a(t)}+\frac{1}{a(t)\left(t-t_{0}+t_{1}\right)} \sqrt{a(t)\left(\left(t-t_{0}+t_{1}\right)^{2} F_{1}(t)-u_{1}^{2}(x)+2 \int^{x} u_{1}(s) d s\right) .}$

Now inserting the expression 4.11 into Eq. 4.7) implies that necessarily

$$
a(t)=\frac{a_{0}}{\left(t-t_{0}+t_{1}\right)^{2}},
$$

with $a_{0} \in \mathbb{R}$. Similarly, inserting the expression 4.11 into Eq. 4.8) allows to obtain the expression $b(t)$ under the form

$$
b(t)=g+\frac{2 b_{0}}{\left(t-t_{0}+t_{1}\right)^{2}},
$$

with $b_{0} \in \mathbb{R}$.

Thus, equation 4.14 gives

$$
\begin{aligned}
h(t, x)=-z_{b}(x)-\frac{b_{0}}{a_{0}}-\frac{g}{a_{0}}( & \left.t-t_{0}+t_{1}\right)^{2} \\
& +\frac{1}{\sqrt{a_{0}}} \sqrt{\left(t-t_{0}+t_{1}\right)^{2} F_{1}(t)-u_{1}^{2}(x)+2 \int^{x} u_{1}(s) d s}
\end{aligned}
$$

The two expressions for $h(t, x)$, namely 4.13 and 4.15 are compatible only if the primitive function associated to $\frac{1}{u_{1}(x)}$ is a logarithmic function of $x$, leading to

$$
u_{1}(x)=\gamma x+\beta,
$$

with $(\beta, \gamma) \in \mathbb{R}^{2}$. Inserting 4.16 into 4.7) gives that

$$
\gamma^{2}+\gamma=a
$$

Now from Eq. 4.14 we can set $-u_{1}^{2}(x)+2 \int^{x} u_{1}(s) d s=C$ in order to have $h(t, x)+z_{b}(x)$ only depending on time $t$ i.e.

$$
\frac{\partial \eta}{\partial x}=0
$$

This property inserted into 4.6 gives $\gamma=1$, and using equation 4.17), $a_{0}=2$.

Finally we have obtained that

$$
u_{1}(x)=x+\beta,
$$

and $h=z_{b}(x)+F_{\eta}(t)$. These two expressions inserted in Eq. 4.5 give

$$
\left\{\begin{array}{l}
z_{b}(x)=\frac{c_{0}}{x+\beta}-b_{0} \\
h(t, x)=\frac{\alpha}{t-t_{0}+t_{1}}-\frac{c_{0}}{x+\beta}
\end{array}\right.
$$

These expressions are valid only if $h(t, x) \geqslant 0$. Thus, the expressions 4.19, $4.20,4.11$ give a proof of Prop 3 in the two-dimensional $(x, z)$ setting (when $\theta=0$ ).

COROLlary 3. If we assume that all the solutions of Eq. 4.7 can be written under the form 4.11), Prop. 3 gives all the solutions of the free surface Euler equations in which $u$ and $v$ do not depend on $z$.

Figure 7 shows the velocity field at time $t=0,0.05,0.1$ and 0.15 second for a given set 

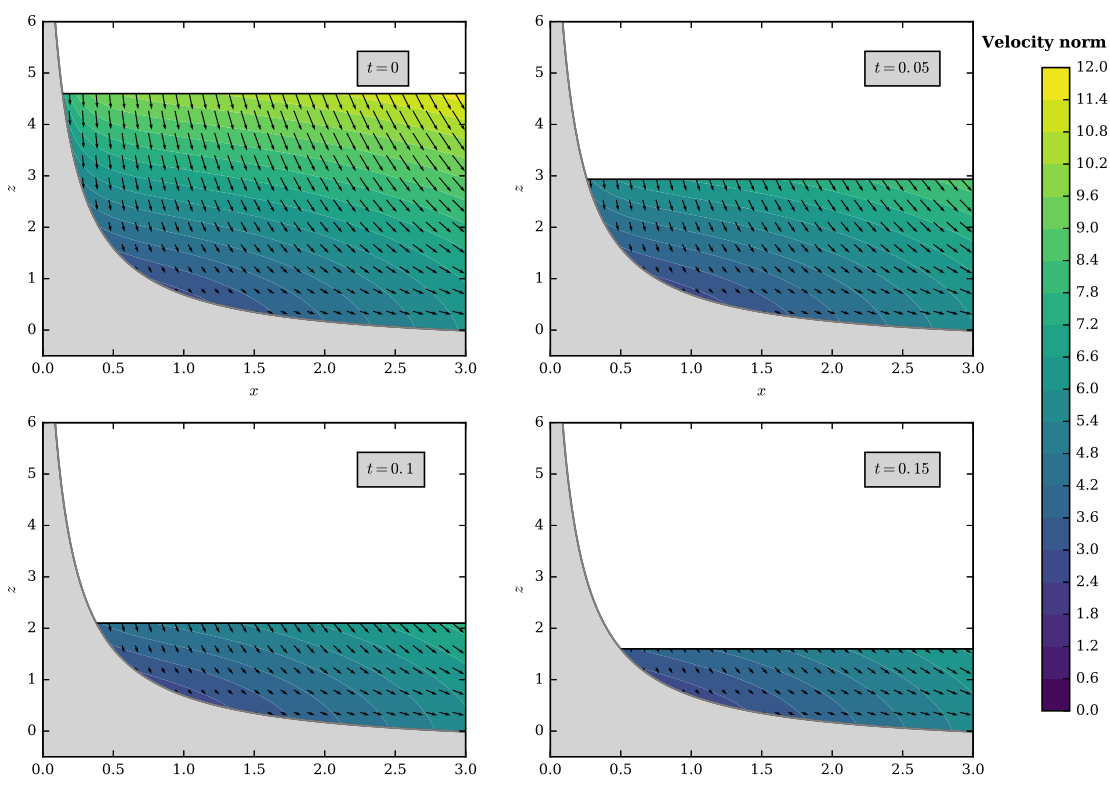

FiguRE 7. Analytical solution for hyperbolic topography (see prop 3): velocity norm and vectors at $t=0,0.05,0.1$ and $0.15 \mathrm{~s}$ in $(x, y=0, z)$ for parameters set to $\alpha=0.5 \mathrm{~m} . \mathrm{s}, \beta=0.1 \mathrm{~m}$, $b_{0}=0.4 \mathrm{~m}, t_{0}-t_{1}=2 \mathrm{~s}, c_{0}=1.2 \mathrm{~m}^{2}$.

of the parameters value ( $\left.\alpha=0.5 \mathrm{~m} . \mathrm{s}, \beta=0.1 \mathrm{~m}, b_{0}=0.4 \mathrm{~m}, t_{0}-t_{1}=2 \mathrm{~s}, c_{0}=1.2 \mathrm{~m}^{2}\right)$. The solution proposed in proposition 3 is not a solution of the hydrostatic formulation of the equation, but we can easily deduce a hydrostatic analytical solution by reversing the direction of the velocity field. This result is described in the following paragraph.

\subsection{Hydrostatic Euler equations}

If we consider the hydrostatic Euler system 2.7$),(2.8)$, the following result holds.

Proposition 4. For some $\left(\alpha, \beta, c_{0}, t_{0}\right) \in \mathbb{R}^{+3},\left(b_{0}, t_{o}\right) \in \mathbb{R}^{2}, t_{1} \in \mathbb{R}_{+}^{*}, \theta \in[0,2 \pi]$, let us consider the function $h, u, v, w$ and $p$ defined for $t \geqslant t_{0}$ by

$$
\begin{aligned}
& h(t, x, y)=\max \left\{0, \alpha f(t)-b_{0}-z b(x, y)\right\}, \\
& u(t, x, y, z)=-(x \cos \theta+y \sin \theta+\beta) \cos \theta / f(t), \\
& v(t, x, y, z)=-(x \cos \theta+y \sin \theta+\beta) \sin \theta / f(t), \\
& w(t, x, y, z)=\left(b_{0}+z\right) / f(t), \\
& p(t, x, y, z)=p^{a, 0}(t)+p^{a, 1}(t, x, y)+g(\eta-z),
\end{aligned}
$$

where $f(t)=t-t_{0}+t_{1}$, the bottom topography given by $z_{b}(x, y)=\frac{c_{0}}{x \cos \theta+y \sin \theta+\beta}-b_{0}$ and $p^{a, 0}(t)$ a given function with also

$$
p^{a, 1}(t, x, y)=-\left((x \cos \theta+y \sin \theta)^{2}+2 \beta(x \cos \theta+y \sin \theta)\right) / f(t)^{2} .
$$

Then $h, u, v, p$ as defined previously satisfy the hydrostatic Euler system (2.7), 2.8) completed with the boundary conditions (2.3), eqrefeq:bottom and 2.5). 

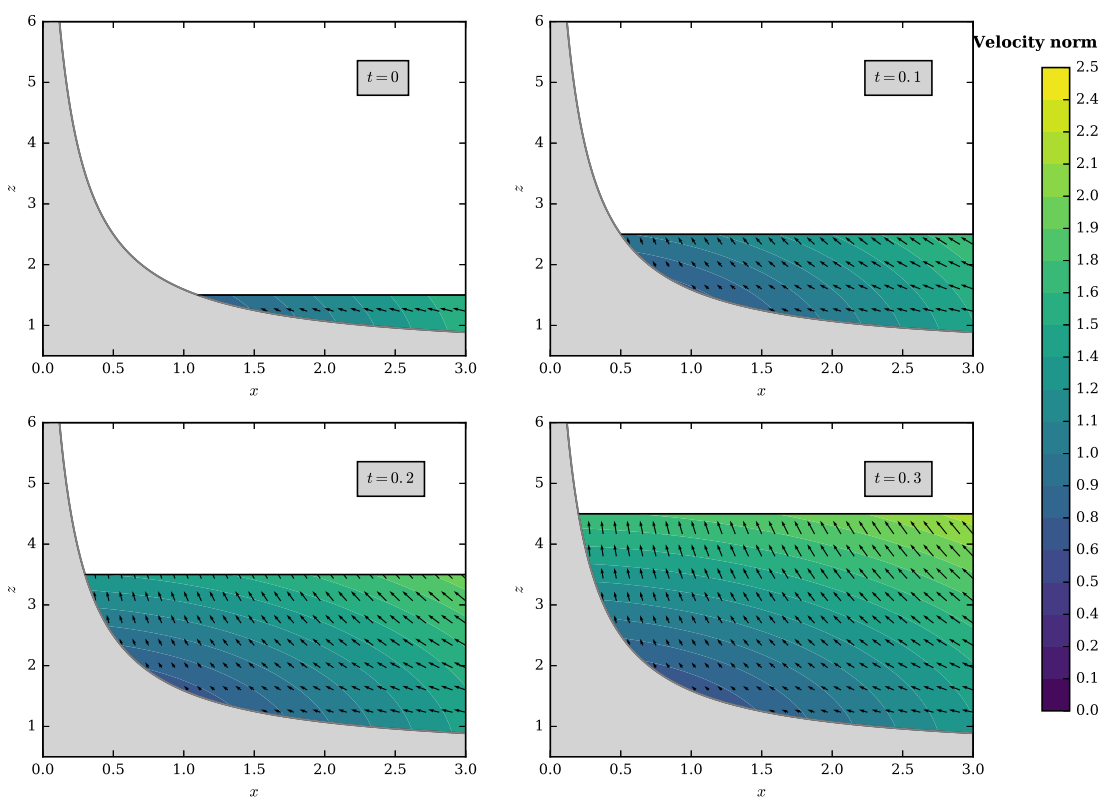

Figure 8. Decreasing bathymetry in $1 / x$ : velocity norm and vectors at $t=0,0.1,0.2$ and $0.3 \mathrm{~s}$ in $(x, y=0, z)$ for the parameters set $\alpha=1 \mathrm{~m} . \mathrm{s}, \beta=0.1 \mathrm{~m}, b_{0}=-1 \mathrm{~m}, t_{0}-t_{1}=2 \mathrm{~s}$, $c_{0}=1.2 \mathrm{~m}^{2}$.

Proof. The main idea of the proof is to observe that the analytical solution proposed in Prop. 3 satisfies

$$
\frac{\partial w}{\partial t}=u \frac{\partial w}{\partial x}+v \frac{\partial w}{\partial y}+w \frac{\partial w}{\partial z}=-\frac{b_{0}+z}{f(t)^{2}} .
$$

It is sufficient to reverse the direction of the components of the velocity to cancel the non hydrostatic part of the third momentum equation. Compared to Prop. 3, with opposite values of $u$ and $v$, the water depth increases in time. We have then to find the pressure term allowing to satisffy exactly the two other momentum equations.

Figure 8 shows the velocity fields at times $t=0,0.1,0.2$ and $0.3 \mathrm{~s}$ for a given set of the parameters value $\left(\alpha=0.5 \mathrm{~m} . \mathrm{s}, \beta=0.1 \mathrm{~m}, b_{0}=0.4 \mathrm{~m}, t_{0}-t_{1}=2 \mathrm{~s}, c_{0}=1.2 \mathrm{~m}^{2}\right)$.

\section{Tank}

We consider in this section that the domain is a cubic tank with lateral artificial boundary conditions. The free surface remains horizontal and decreases linearly with time. With this hypothesis, we can exhibit some exact solution of the Euler equations, under the hydrostatic assumption or in the non hydrostatic case.

These analytical solutions permit to test the good implementation of the boundary conditions on artificial boundaries in a simulation code. Indeed, the solutions are exact in all the domain, including the boundaries and we can then compute all the derivatives of theses solutions at the boundaries. For example, if we want to test a condition such that $U \cdot n=\psi$ on a boundary, $\psi$ being a given function, we can impose $U \cdot n=U_{a} \cdot n$ where $U_{a}$ is the analytical solution which is given here. 


\subsection{Euler equations with hydrostatic hypothesis}

Considering the hydrostatic Euler system given in paragraph 2.3 in a tank such that $(x, y) \in[-L / 2, L / 2]^{2}$. The two following propositions hold.

Proposition 5. For some $t_{0} \in \mathbb{R}, t_{1} \in \mathbb{R}_{+}^{*},(\alpha, \beta) \in \mathbb{R}_{+}^{2}$ such that $\alpha \beta>$ L, let us consider the functions $h, u, v, w, p$ defined for $t \geqslant t_{0}$ by

$$
\begin{aligned}
& h(t, x, y)=\alpha f(t), \\
& u(t, x, y, z)=\beta\left(\left(z-z_{b}\right)-\frac{\alpha}{2} f(t)\right)+f(t)\left(x \cos ^{2} \theta+y \sin ^{2} \theta\right), \\
& v(t, x, y, z)=\beta\left(\left(z-z_{b}\right)-\frac{\alpha}{2} f(t)\right)+f(t)\left(x \cos ^{2} \theta+y \sin ^{2} \theta\right), \\
& w(t, x, y, z)=f(t)\left(z_{b}-z\right), \\
& p(t, x, y, z)=p^{a}(t, x, y)+g(\eta-z),
\end{aligned}
$$

where $f(t)=1 /\left(t-t_{0}+t_{1}\right), p^{a}(t, x, y)=p^{a, 1}(t)$, with $p^{a, 1}(t)$ a given function and with a flat bottom $z_{b}(x, y)=z_{b, 0}=$ cst.

Then $h, u, v, w, p$ as defined previously satisfy the 3d hydrostatic Euler system (2.7)(2.8) completed with the boundary conditions (2.3), 2.4) and (2.5). The appropriate boundary conditions for $x, y= \pm L / 2$ are also determined by the expressions of $h, u, v, w$ given above.

The vertical acceleration - corresponding to the right hand side of $(2.6)$ - in this analytical solution is equal to $2 z /\left(t-t_{0}+t_{1}\right)^{2}<2 h^{3} / \alpha^{2}$. The hydrostatic hypothesis is justified only for values of $h$ small enough that corresponds to a shallow water regime.

Proof. First, we observe that

$$
\frac{\partial h}{\partial t}=\frac{-\alpha}{\left(t-t_{0}+t_{1}\right)^{2}},
$$

and

$$
\frac{\partial(h u)}{\partial x}=\frac{\alpha \sin ^{2}(\theta)}{\left(t-t_{0}+t_{1}\right)^{2}} \quad, \quad \frac{\partial(h v)}{\partial y}=\frac{\alpha \cos ^{2}(\theta)}{\left(t-t_{0}+t_{1}\right)^{2}} .
$$

Then, taking the sum of the last three terms, the mass conservation equation is satisfied. For the second equation, we need to verify that

$$
\frac{\partial u}{\partial t}+\frac{\partial u^{2}}{\partial x}+\frac{\partial(u v)}{\partial y}+\frac{\partial(u w)}{\partial z}+g \frac{\partial\left(h+z_{b}\right)}{\partial x}=0,
$$

We can observe that $u=v$ moreover $h$ and $z_{b}$ do not depend on $x$. Then we have to check

$$
\frac{\partial u}{\partial t}+\frac{\partial u^{2}}{\partial x}+\frac{\partial u^{2}}{\partial y}+\frac{\partial u w}{\partial z}=0
$$

with $w$ given thanks to the incompressibility condition by

$$
w(t, x, y, z)=-\frac{\partial}{\partial x} \int_{z_{b}}^{z} u d z-\frac{\partial}{\partial y} \int_{z_{b}}^{z} v d z .
$$

Since we have

$$
\begin{gathered}
\frac{\partial u}{\partial t}=-\frac{\alpha \beta}{2} f^{\prime}(t)+f^{\prime}(t)\left(x \cos ^{2}(\theta)+y \sin ^{2}(\theta)\right), \\
\frac{\partial u^{2}}{\partial x}=2 u f(t) \cos ^{2}(\theta) \quad, \quad \frac{\partial u^{2}}{\partial y}=2 u f(t) \sin ^{2}(\theta),
\end{gathered}
$$




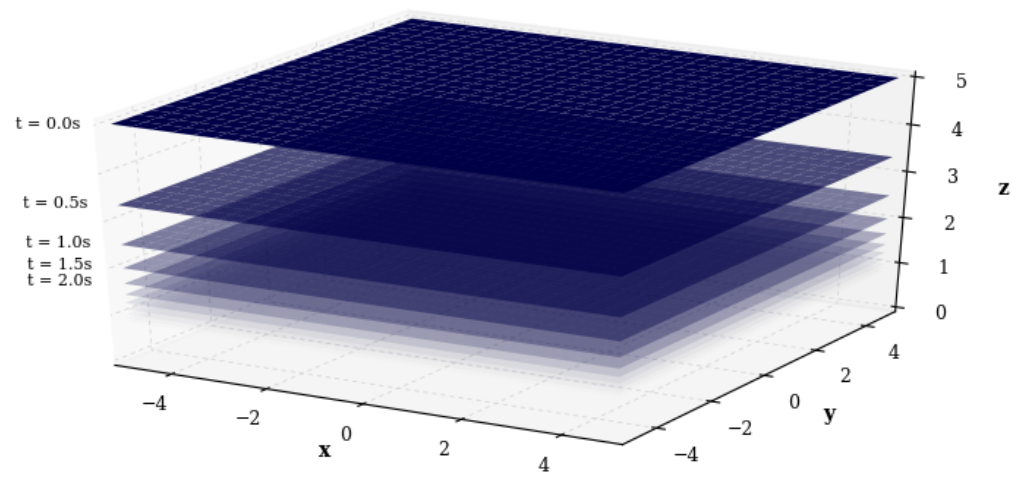

FIgURE 9. Draining of a tank: free surface every $0.5 \mathrm{~s}$ from initial time, for parameters set to $\alpha=5 \mathrm{~m} . \mathrm{s}, \beta=0 \mathrm{~s}^{-1}, t_{0}=1 \mathrm{~s}, t_{1}=1 \mathrm{~s}$ and $L=10 \mathrm{~m}$.

and

$$
\frac{\partial(u w)}{\partial z}=-u f(t)+\beta f(t)\left(z_{b}-z\right)
$$

then, using the property $f^{\prime}(t)=-f^{2}(t)$ and taking the sum of Eqs. (5.3), (5.4), (5.5), we can easily verify that Eq. $(5.2)$ holds true since

$$
\begin{aligned}
\frac{\partial u}{\partial t}+\frac{\partial u^{2}}{\partial x}+\frac{\partial u^{2}}{\partial y}+\frac{\partial u w}{\partial z} & =f(t)\left(\frac{\alpha \beta}{2} f(t)-f(t)\left(x \cos ^{2}(\theta)+y \sin ^{2}(\theta)\right)-\beta\left(z-z_{b}\right)+u\right) \\
& =0 .
\end{aligned}
$$

REMARK 2. Notice that the solution proposed in Prop.5 can be written in the $2 d(x, z)$ case by simply choosing $\theta=0$.

Figure 9 represents the free surface elevation at different time instants $(t=$ $0.0 s, 0.5 s, 1 s, 1.5 s, 2.0 s)$ for $\alpha=5 \mathrm{~m} . \mathrm{s}, \beta=0 \mathrm{~s}^{-1} L=10 \mathrm{~m}, t_{0}=0 \mathrm{~s}$ and $t_{1}=1 \mathrm{~s}$. The form of the free surface elevation is similar in Props. 5 and 6 .

\subsection{Euler equations without hydrostatic hypothesis}

Considering the Euler system (2.1)-(2.2) in a tank such that $(x, y) \in[-L / 2, L / 2]^{2}$. The following proposition holds.

Proposition 6. For some $\left.\alpha \in \mathbb{R}_{+},\left(t_{0}, \gamma, \delta\right) \in \mathbb{R}^{3}, t_{1} \in \mathbb{R}_{+}^{*}, \theta \in\right] 0,2 \pi[/\{\pi\}$ let us consider the functions $h, u, v, w, p$ defined for $t \geqslant t_{0}$ by

$$
\begin{aligned}
& h(t, x, y)=\alpha f(t), \\
& u(t, x, y, z)=f(t)(x \cos \theta+y \sin \theta)+\gamma\left(z-z_{b}\right)+\delta, \\
& v(t, x, y, z)=f(t)(x \cos \theta+y \sin \theta) \tan \frac{\theta}{2}+\frac{1-\cos \theta}{\sin \theta} \gamma\left(z-z_{b}\right)-\frac{\delta}{\tan \theta}, \\
& w(t, x, y, z)=f(t)\left(z_{b}-z\right), \\
& p(t, x, y, z)=p^{a, 0}(t)+g\left(h-\left(z-z_{b}\right)\right)+\left(h^{2}-\left(z-z_{b}\right)^{2}\right) f(t)^{2},
\end{aligned}
$$

where $f(t)=1 /\left(t-t_{0}+t_{1}\right), h_{0}=h\left(t_{0}, x, y\right)=\alpha / t_{1}, p^{a, 0}(t)$ a given function and with $a$ flat bottom $z_{b}(x, y)=z_{b, 0}=$ cst.

Then $h, u, v, w, p$ as defined previously satisfy the $3 d$ Euler system (2.1)-(2.2) completed 
20 M.-O. Bristeau, B. Di Martino, A. Mangeney, J. Sainte-Marie and F. Souillé

with the boundary conditions 2.3, (2.4) and 2.5). The appropriate boundary conditions for $x, y= \pm L / 2$ are also determined by the expressions of $h, u, v, w, p$ given above.

Notice that the velocities $u, v$ and $w$ are linear in $x, y$ or $z$.

Proof. The results is based on two observations. First, the function $f$ is such that $f^{\prime}(t)=-f^{2}(t)$. Second, using the trigonometric formula

$$
\cos \theta=\frac{1-\tan ^{2}\left(\frac{\theta}{2}\right)}{1+\tan ^{2}\left(\frac{\theta}{2}\right)} \quad, \quad \sin \theta=\frac{2 \tan \left(\frac{\theta}{2}\right)}{1+\tan ^{2}\left(\frac{\theta}{2}\right)},
$$

we can observe that

$$
\cos \theta+\sin \theta \tan \left(\frac{\theta}{2}\right)=1
$$

This formula $(5.6)$ will be used many times in the proof.

We can easily compute that

$$
\frac{\partial u}{\partial x}=f(t) \cos \theta, \quad \frac{\partial v}{\partial y}=f(t) \sin \theta \tan \frac{\theta}{2}, \quad \frac{\partial w}{\partial z}=-f(t) .
$$

leading to $\nabla \cdot U=0$ using formula $(5.6)$. For the first momentum equation, we have to verify that the solution is such that

$$
\frac{\partial u}{\partial t}+u \frac{\partial u}{\partial x}+v \frac{\partial u}{\partial y}+w \frac{\partial u}{\partial z}+\frac{1}{\rho_{0}} \frac{\partial p}{\partial x}=0 .
$$

We can observe that $\frac{\partial p}{\partial x}=0$ since the functions $f, z_{b}$, and $h$ do not depend on $x$. For the other terms, we have

$$
\begin{gathered}
\frac{\partial u}{\partial t}=-(x \cos \theta+y \sin \theta) f^{2}(t), \quad u \frac{\partial u}{\partial x}=u f(t) \cos \theta \\
v \frac{\partial u}{\partial y}=v f(t) \sin \theta, \quad w \frac{\partial u}{\partial z}=\gamma f(t)\left(z_{b}-z\right) .
\end{gathered}
$$

Then,

$$
\begin{gathered}
u \frac{\partial u}{\partial x}+v \frac{\partial u}{\partial y}+w \frac{\partial u}{\partial z}=f(t)\left(u\left(2 \cos \theta+\sin \theta \tan \left(\frac{\theta}{2}\right)-1\right)+v \cos \theta+\gamma\left(z_{b}-z\right)\right) \\
=f(t)\left[f(t) x \cos ^{2} \theta+f(t) y \sin \theta \cos \theta+\gamma\left(z-z_{b}\right) \cos \theta+\delta \cos \theta+f(t) x \cos \theta \sin \theta \tan \left(\frac{\theta}{2}\right)\right. \\
\left.+f(t) y \sin ^{2} \theta \tan \left(\frac{\theta}{2}\right)+(1-\cos \theta) \gamma\left(z-z_{b}\right)-\delta \cos \theta+\gamma\left(z_{b}-z\right)\right] .
\end{gathered}
$$

We can observe that the terms depending on $\delta$ and $\gamma$ are equal to 0 . For the terms in $x$, we have

$$
f^{2}(t)\left(\cos \theta\left(\cos \theta+\sin \theta \tan \left(\frac{\theta}{2}\right)\right)\right)=f^{2}(t) \cos \theta
$$

and for the terms in $y$

$$
f^{2}(t) \sin \theta\left(\cos \theta+\sin \theta \tan \left(\frac{\theta}{2}\right)\right)=f^{2}(t) \sin \theta .
$$

Combining all these results, we conclude that the first momentum equation is verified. The computation for the second momentum equation is very similar. For the third 


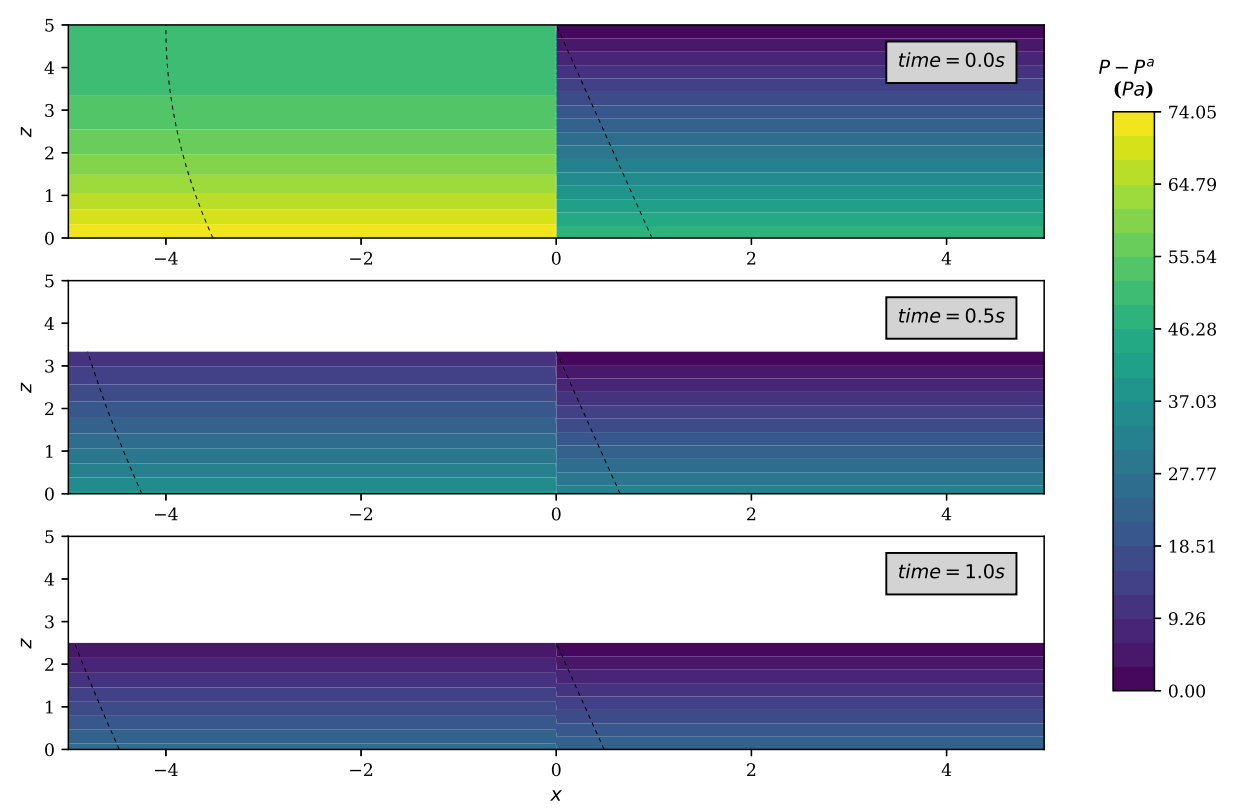

FIGURE 10. Analytical solution for the draining of a tank (see prop. 6 non-hydrostatic pressure (left, $x<0$ ) and hydrostatic pressure (right $x>0$ ), at $t=0,0.5$ and $1 \mathrm{~s}$, in $(x, y=0, z)$ plane with parameters set to $\gamma=0 \mathrm{~s}^{-1}, \delta=0 \mathrm{~m} \cdot \mathrm{s}^{-1}, \theta=0, \alpha=5 \mathrm{~m} . \mathrm{s}, t_{0}=1 \mathrm{~s}, t_{1}=1 \mathrm{~s}$, $p^{a}=0 \mathrm{~m}^{2} \cdot \mathrm{s}^{-2}, z_{b}=0 \mathrm{~m}$ and $L=10 \mathrm{~m}$. Dashed line represents pressure profile.

momentum equation, the computation is easier since $w$ does not depend on $x$ and $y$. We have only to compute

$$
\frac{\partial w}{\partial t}=-f^{2}\left(z_{b}-z\right), w \frac{\partial w}{\partial z}=-f^{2}(t)\left(z_{b}-z\right), \frac{\partial p}{\partial z}=-g+2 f^{2}(t)\left(z_{b}-z\right),
$$

and the result is proved.

REMARK 3. If we consider a viscous fluid governed by the Navier-Stokes equations, the stress tensor $\Sigma=\mu\left(\nabla U+(\nabla U)^{T}\right)$ (where $\mu$ is the viscosity coefficient (unit : Pa.s)) is then equal to zero and the proposed solution is also a solution of the NavierStokes equations. On the computational point of view, it is an interesting property since simulating such a solution of the Navier-Stokes system, all the components of the stress tensor $\Sigma$ have to remain zero.

For $\theta=0$ the flow is only in the direction of $x$ and we can consider only a two dimensional version of the solution in $(x, z)$ coordinates. Nevertheless, the angle $\theta$ does not represent the direction of the flow in the $(x, y)$ plane.

In Figure 10 we present a vertical profile of the analytical solution. With the given data, $h(t)=5 /(t+1)$, we have at initial time, $h(0)=5 \mathrm{~m}$ and at $t=1 \mathrm{~s}, h(1)=\frac{5}{2} \mathrm{~m}$. For $x>0$ (right part of the figure), the pressure is hydrostatic and increases linearly with the depth whereas for $x<0$ (left part of the figure), the pressure includes a non hydrostatic component (quadratic in $z-z_{b}$ ). 
5.3. Non hydrostatic Euler equations with passive tracer

Considering the Euler system given in Section 2.1 by equations 2.1)-(2.2) including the tracer equation (2.11) and formulated in a tank such that $(x, y) \in[-L / 2, L / 2]^{2}$, the following proposition holds.

Proposition 7. For some $\alpha \in \mathbb{R}_{+}, t_{0} \in \mathbb{R}, t_{1} \in \mathbb{R}_{+}^{*}, \phi_{0} \in \mathbb{R}, \theta \in[0,2 \pi] /\{\pi\}$ let us consider the functions $h, u, v, w, p, \phi$ defined for $t \geqslant t_{0}$ by

$$
\begin{aligned}
& h(t, x, y)=\alpha f(t), \\
& u(t, x, y, z)=f(t)(x \cos \theta+y \sin \theta), \\
& v(t, x, y, z)=f(t)(x \cos \theta+y \sin \theta) \tan \frac{\theta}{2}, \\
& w(t, x, y, z)=f(t)\left(z_{b}-z\right), \\
& p(t, x, y, z)=p^{a, 0}(t)+g\left(h-\left(z-z_{b}\right)\right)+\left(h^{2}-\left(z-z_{b}\right)^{2}\right) f(t)^{2}, \\
& \phi(t, x, y, z)=\frac{\phi_{0}}{L}(x \cos \theta+y \sin \theta) \frac{z-z_{b}}{h_{0}},
\end{aligned}
$$

where $f(t)=1 /\left(t-t_{0}+t_{1}\right), h_{0}=h\left(t_{0}, x, y\right)=\alpha / t_{1}, p^{a, 0}(t)$ a given function and with $a$ flat bottom topography $z_{b}(x, y)=z_{b, 0}=$ cst.

Then $h, u, v, w, p$ and $\phi$ satisfy the $3 d$ Euler system (2.1)-(2.2) completed with the boundary conditions (2.3), 2.4 , 2.5) and the tracer equation (2.11). The appropriate boundary conditions for $x, y= \pm L / 2$ are also determined by the expressions of $h, u, v, w, p$ given above.

Proof. The proof relies on very simple computations since it is enough to verify that these functions are solutions of the equations of Euler given in paragraph 2.1. The computations are very similar to those detailed in the proof of Prop. 6 for the mass and momentum equations. We focus here only on the transport equation (2.11) and we use mainly the property presented in $(5.6)$. We have

- $\frac{\partial \phi}{\partial t}=0$,

- $u \frac{\partial \phi}{\partial x}=\frac{\phi_{0}}{L} \cos \theta \frac{z-z_{b}}{h_{0}} f(t)(x \cos \theta+y \sin \theta)$,

- $v \frac{\partial \phi}{\partial y}=\frac{\phi_{0}}{L} \sin \theta \frac{z-z_{b}}{h_{0}} f(t)(x \cos \theta+y \sin \theta) \tan \left(\frac{\theta}{2}\right)$,

- $w \frac{\partial \phi}{\partial z}=\frac{\phi_{0}}{L} \frac{z-z_{b}}{h_{0}} f(t)(x \cos \theta+y \sin \theta)$.

Then

$$
\begin{aligned}
\frac{\partial \phi}{\partial t}+u \frac{\partial \phi}{\partial x}+v \frac{\partial \phi}{\partial y}+ & w \frac{\partial \phi}{\partial z}= \\
& \frac{\phi_{0}}{L} \frac{z-z_{b}}{h_{0}} f(t)\left(\cos \theta+\sin \theta \tan \left(\frac{\theta}{2}\right)-1\right)(x \cos \theta+y \sin \theta)=0 .
\end{aligned}
$$

COROLlary 4. The analytical solution depicted in Prop. 7 is also solution of the Euler system with a passive tracer - having a concentration $\phi$ - governed by a transport diffusion equation

$$
\frac{\partial \phi}{\partial t}+U \cdot \nabla \phi-\mu_{\phi} \Delta \phi=0
$$




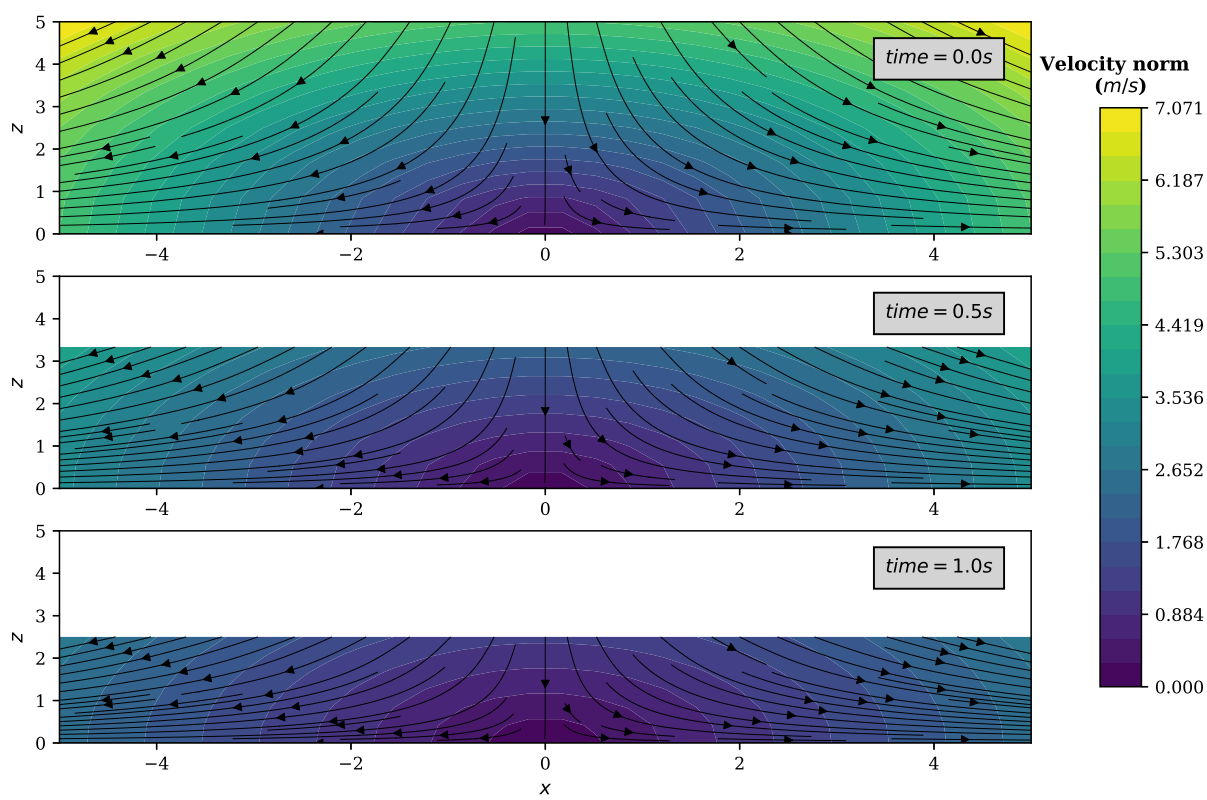

FIGURE 11. Draining of a tank (non-hydrostatic case): velocity norm and streamlines at $t=0,0.5,1.0 \mathrm{~s}$, in $(x, y=0, z)$ slice plane for parameters set to $\theta=0, \alpha=\mathrm{m} . \mathrm{s}, t_{1}=1 \mathrm{~s}$, $p^{a}=0 \mathrm{~m}^{-2} \cdot \mathrm{s}^{-2}, z_{b}=0 \mathrm{~m}$ and $L=10 \mathrm{~m}$.
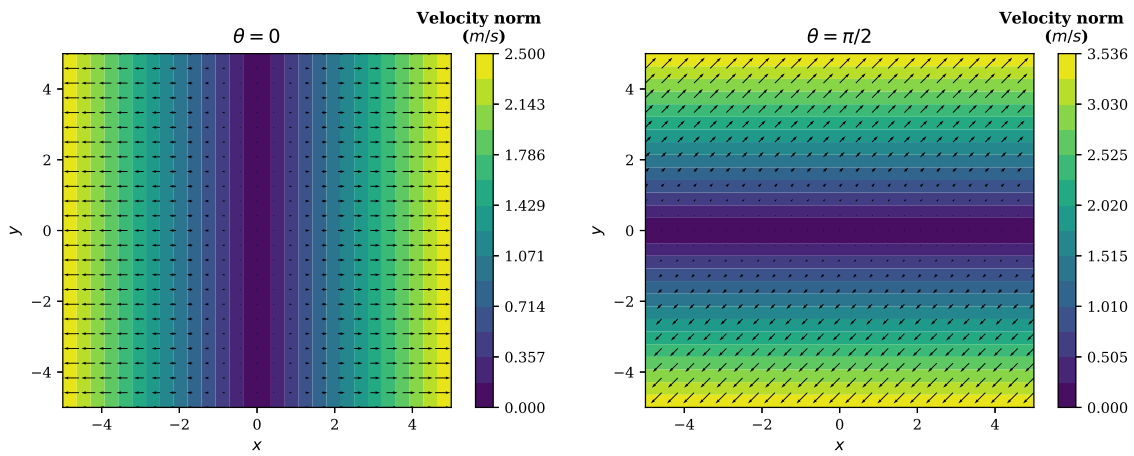

FiguRE 12. Draining of a tank (non-hydrostatic case): velocity norm and vectors at initial time in $\left(x, y, z=h_{0} / 2\right)$ slice plane with $\alpha=5 \mathrm{~m} . \mathrm{s}, t_{1}=1 \mathrm{~s}, p^{a}=0 \mathrm{~m}^{2} \cdot \mathrm{s}^{-2}, z_{b}=0 \mathrm{~m}$ and $L=10 \mathrm{~m}$.

In this case, we need to consider additional boundary conditions given by

$$
\left.\mu_{\phi} \nabla \phi\right|_{s} \cdot \mathbf{n}_{s}=\mu_{\phi} \phi_{0}(x \cos \theta+y \sin \theta) /\left(L h_{0}\right)
$$

and

$$
\left.\mu_{\phi} \nabla \phi\right|_{b} \cdot \mathbf{n}_{b}=-\mu_{\phi} \phi_{0}(x \cos \theta+y \sin \theta) /\left(L h_{0}\right) .
$$

where subscript $s$ (resp. b) refers to the free surface (resp. bottom).

\section{Acknowledgement}

The work presented in this paper was supported in part by the ANR Mimosa (ANR14-CE01-0012) and ERC contract ERC-CG-2013-PE10-617472 SLIDEQUAKES. The 
authors acknowledge the Inria Project Lab "Algae in Silico" for its financial support. We also thank the reviewers who allowed us to improve the presentation of this document.

\section{REFERENCES}

Allgeyer, S., Bristeau, M.-O., Froger, D., Hamouda, R., Jauzein, V., Mangeney, A., Sainte-Marie, J., Souillé, F. \& Vallée, M. 2019 Numerical approximation of the 3d hydrostatic Navier-Stokes system with free surface. ESAIM: M2AN 53 (6), 1981-2024.

Boulanger, A.C., Bristeau, M.O. \& Sainte-Marie, J. 2013 Analytical solutions for the free surface hydrostatic Euler equations. Communications in Mathematical Sciences 11, 993-1010.

Brenier, Y. 1999 Homogeneous hydrostatic flows with convex velocity profiles. Nonlinearity $12(3), 495-512$.

Fernandez-Nieto, E. D., Garres-Diaz, J., Mangeney, A. \& Narbona-Reina, G 2016 A multilayer shallow model for dry granular flows with the mu(I) rheology: Application to granular collapse on erodible beds. J. Fluid. Mech. 798, 643-681.

FRESHKISS3D 2017 home page. http://freshkiss3d.gforge.inria.fr

GRENIER, E. 1999 On the derivation of homogeneous hydrostatic equations. ESAIM: M2AN 33 (5), 965-970.

MacDonald, I., Baines, M.J., Nichols, N.K. \& Samuels, P.G. 1995a Comparisons of some steady state Saint-Venant solvers for some test problems with analytic solutions. Tech. Rep. 3/95. Dept of Mathematics, University of Reading, http://www.reading.ac.uk/maths/research/maths-numanalrpts.aspx\#1995.

MacDonald, I., Baines, M.J., Nichols, N.K. \& Samuels, P.G. 1995b Steady open channel test problems with analytic solutions. Tech. Rep. 2/95. Dept of Mathematics, University of Reading, http://www.reading.ac.uk/maths/research/maths-numanalrpts.aspx\#1995.

Matskevich, N.A. \& Chubarov, L.B. 2019 Exact Solutions to Shallow Water Equations for a Water Oscillation Problem in an Idealized Basin and Their Use in Verifying Some Numerical Algorithms. Numerical Analysis and Applications 19, 234-250.

ThACKer, W. C. 1981 Some exact solutions to the non-linear shallow-water wave equations. $J$. Fluid Mech. 107, 499-508. 